\title{
Formation of Specific Monosynaptic Connections between Muscle Spindle Afferents and Motoneurons in the Mouse
}

\author{
Simon C. Mears and Eric Frank \\ Department of Neurobiology, University of Pittsburgh School of Medicine, Pittsburgh, Pennsylvania 15261
}

\begin{abstract}
In adult vertebrates, sensory neurons innervating stretchsensitive muscle spindles make monosynaptic excitatory connections with specific subsets of motoneurons in the spinal cord. Spindle afferents (la fibers) make the strongest connections with motoneurons supplying the same (homonymous) muscle but make few or no connections with motoneurons supplying antagonistic or functionally unrelated muscles. In lower vertebrates these connections are specific from the time they first are formed, but there is comparatively little information about how these reflex connections form in mammals. We therefore studied the pattern of these synaptic connections during postnatal development in mice. Intracellular recordings were made from identified hindlimb motoneurons in an isolated spinal cord preparation, and monosynaptic inputs from la fibers in identified hindlimb muscle nerves were measured at different times during the first
\end{abstract}

postnatal week. The pattern of connections was specific throughout this period. la fibers made strong connections with homonymous motoneurons but only weak connections with antagonistic motoneurons at every time point examined, from P0 through P7. Even when muscle nerves were stimulated at only $0.1 \mathrm{~Hz}$, the pattern of connections was still highly specific, arguing against a special subpopulation of labile inappropriate connections. The absence of appreciable rearrangements in the pattern of these connections during the first postnatal week is, therefore, analogous to the situation in lower vertebrates, suggesting that mechanisms responsible for establishing this specificity have been conserved during evolution.

Key words: synaptogenesis; synaptic specificity; motoneurons; muscle spindle afferents; la fibers; spinal cord; stretch reflex
For the nervous system to function correctly, specific patterns of synaptic connections must be established between appropriate groups of neurons. A commonly accepted paradigm for achieving this specificity is that chemoaffinity mechanisms are used to establish the initial pattern of connections, and then these connections are remodeled during subsequent development to refine the connectivity further (Goodman and Shatz, 1993). Coordinated electrical activity between pre- and postsynaptic partners can play a critical role in the refinement process.

An alternative strategy used in other developmental systems is that chemoaffinity mechanisms are sufficient to produce a highly precise pattern of connections. In these systems, which have received the most attention in lower vertebrates and invertebrates, synaptic rearrangements are not seen. One example is the set of connections between muscle spindle sensory axons (Ia fibers) and spinal motoneurons, the synaptic pathway that mediates the simple stretch reflex. Ia afferents from one muscle make strong monosynaptic excitatory synapses with motoneurons supplying the same and synergistic muscles, but Ia afferents make few if any direct connections with motoneurons supplying antagonistic muscles. Studies in the frog and chick have shown that these monosynaptic connections form in a precise, adult-like pattern from the outset (Frank and Westerfield, 1983; Lee et al., 1988). Furthermore, coordinated electrical activity in the sensory and

\footnotetext{
Received Jan. 10, 1997; accepted Feb. 10, 1997.

This research was supported by National Institutes of Health Grant NS24373 to E.F. We thank Ms. Xiaoping Chen for her excellent technical assistance.

Correspondence should be addressed to Dr. Eric Frank, Department of Neurobiology, BST W1452, University of Pittsburgh School of Medicine, 3500 Terrace Street, Pittsburgh, PA 15261.

Copyright (C) 1997 Society for Neuroscience $\quad 0270-6474 / 97 / 173128-08 \$ 05.00 / 0$
}

motor neurons is not required for these connections to develop correctly (Frank and Jackson, 1986; Mendelson and Frank, 1991).

The strategy used in establishing this synaptic pathway in mammals is unclear. On the basis of the results from lower vertebrates, one might expect the pattern to be rigidly determined with little or no rearrangement. On the other hand, mechanisms to refine the initial pattern might have appeared during evolution. The experimental evidence is neither extensive nor definitive. Early electrophysiological experiments showed that the pattern of these connections in fetal (Naka, 1964a,b) and neonatal (Eccles et al., 1963) cats was, for the most part, similar to the adult pattern, suggesting that rearrangements were minimal.

More recently, studies in rats (Seebach and Ziskind-Conhaim, 1994) and humans (Myklebust and Gottlieb, 1993) have demonstrated significant differences between neonatal and adult patterns of reflex connections. It is unclear, however, whether polysynaptic pathways were contributing to the reflex responses that were observed. To address this question further, we have examined the development of these reflex connections in mice, limiting our analysis only to those synaptic potentials with the shortest latencies, thereby focusing on monosynaptic connections of Ia afferents. Both the patterns and amplitudes of these potentials were invariant during the first postnatal week, and the pattern was similar to that described in other adult vertebrates. These results suggest that in mice, as in lower vertebrates, the monosynaptic connections underlying the stretch reflex are probably not extensively rearranged after birth.

\section{MATERIALS AND METHODS}

Animals. Embryos and neonates were obtained from time-dated pregnant Swiss Webster mice (Hilltop Farms, Scottdale, PA). E0 was defined as the day the vaginal plug was first observed and P0 as the first $24 \mathrm{hr}$ period 
after birth. Birth typically occurred between E18 and E19. Embryonic mice were removed from mothers anesthetized with methoxyflurane (Metofane from Pitman-Moore). Then the mother was killed with an overdose of anesthetic.

Dissection. Embryonic or neonatal mice were anesthetized on ice, decapitated, skinned, perfused with cold saline, and eviscerated. Further dissection was performed in recirculating oxygenated $\left(95 \% \mathrm{O}_{2} / 5 \% \mathrm{CO}_{2}\right)$ saline at room temperature $\left(\sim 22^{\circ} \mathrm{C}\right)$ in a SYLGARD-coated dissection dish (Dow Corning, Corning, NY). Saline contained (in mM): $\mathrm{NaCl} 127$, $\mathrm{KCl} 1.9, \mathrm{KH}_{2} \mathrm{PO}_{4} 1.2, \mathrm{CaCl}_{2} 2, \mathrm{MgSO}_{4} 1, \mathrm{NaHCO}_{2} 26$, and dextrose 16.6, $\mathrm{pH}$ 7.4. After dorsal and ventral laminectomy, the spinal cord was isolated and hemisected with fine dissecting scissors. The obturator, quadriceps, and saphenous nerves in one hindlimb were dissected in continuity with the spinal cord. In most cases small nerves to separate heads of the quadriceps muscle branched at one point and were isolated individually. The cord was positioned with its cut medial surface exposed and perfused with oxygenated saline, which was warmed gradually to $30^{\circ} \mathrm{C}$. Nerves were stimulated via individual glass suction electrodes adjusted such that nerve lengths outside the electrodes were approximately equal, minimizing differences in peripheral conduction times of Ia afferents in different nerves.

Intracellular electrophysiology. Motoneurons were impaled with beveled glass micropipettes $\left(90-180 \mathrm{M} \Omega\right.$ ) filled with $2 \mathrm{M} \mathrm{K}^{+}$methylsulfate with $0.5 \%$ fast green added to increase the visibility of the tip. Motoneurons were identified by antidromic activation. To determine the minimum resting potential required for reliable measurement of synaptic input, we compared the amplitude of homonymous synaptic inputs with resting potentials. For resting potentials more negative than $-40 \mathrm{mV}$ there was no obvious correlation ( $r=0.028$ and $p=0.71$, Fisher's $r$-to- $z$ test), so $-40 \mathrm{mV}$ was used as the criterion value.

Nerves were stimulated with square pulses of $0.2 \mathrm{msec}$ duration at supramaximal levels $(7 \mathrm{~V})$, and the resulting synaptic potentials were recorded digitally at $10 \mathrm{kHz}$. Potentials were averaged on-line and stored on a hard disk for subsequent analysis. If the synaptic input caused orthodromic activation, a lower stimulation intensity was used (see Mendelson and Frank, 1991). Homonymous synaptic potentials often were obscured by the antidromic spike (Frank and Westerfield, 1982), for which the threshold is similar to those of Ia afferents. In these cases the stimulus was reduced below this threshold. Because not all Ia afferents were stimulated, this resulted in an underestimate of the true homonymous monosynaptic input. Nevertheless, homonymous EPSPs were still several-fold larger than short-latency input from antagonistic muscle afferents. For the first series of experiments, nerves were stimulated at $\sim 1 \mathrm{~Hz}$, and each potential was averaged 5-20 times on-line. To examine the effects of stimulation frequency, we performed a second series of experiments with stimulus frequencies of 1 and 0.1 $\mathrm{Hz}$. Synaptic potentials were averaged 60 times at $1 \mathrm{~Hz}$ and 15 times at $0.1 \mathrm{~Hz}$.

EPSP analysis. To measure only the monosynaptic Ia component of each synaptic potential, we fit each averaged trace with a standardized monosynaptic EPSP recorded from the same preparation (Sah and Frank, 1984; Mendelson and Frank, 1991). Three examples of this analysis are shown in Figure 1. A homonymous synaptic potential uncontaminated by obvious later components was selected and designated as the monosynaptic model for each animal. The amplitude of the monosynaptic component was measured from the baseline to the peak. Using a computer, we superimposed this model trace over traces to be analyzed and scaled it under software control so that its rising phase during the first few milliseconds matched that of the EPSP being analyzed. The amplitude of the scaled model then gave a measure of the amplitude of the monosynaptic portion of the test EPSP. To avoid experimenter bias, we concealed the identities of the peripheral nerve and motoneuron during the analysis. Because of the similarity of conduction times of Ia afferents in the obturator and quadriceps muscle nerves (see next paragraph), it was possible to use a single monosynaptic model for analyzing all synaptic potentials in each preparation.

Measurement of peripheral conduction times. The peripheral conduction time of sensory impulses was measured by recording extracellular compound dorsal root potentials (CDRPs) in each preparation after intracellular recordings were completed. Dorsal and ventral roots were cut near their entry sites into the spinal cord, and the spinal cord was removed. Lumbar dorsal roots were drawn sequentially into a glass suction electrode for AC recording, and each peripheral nerve was stimulated individually. Most potentials were produced by stimulating supramaximally at $7 \mathrm{~V}$ for $0.2 \mathrm{msec}$. The latency of the earliest

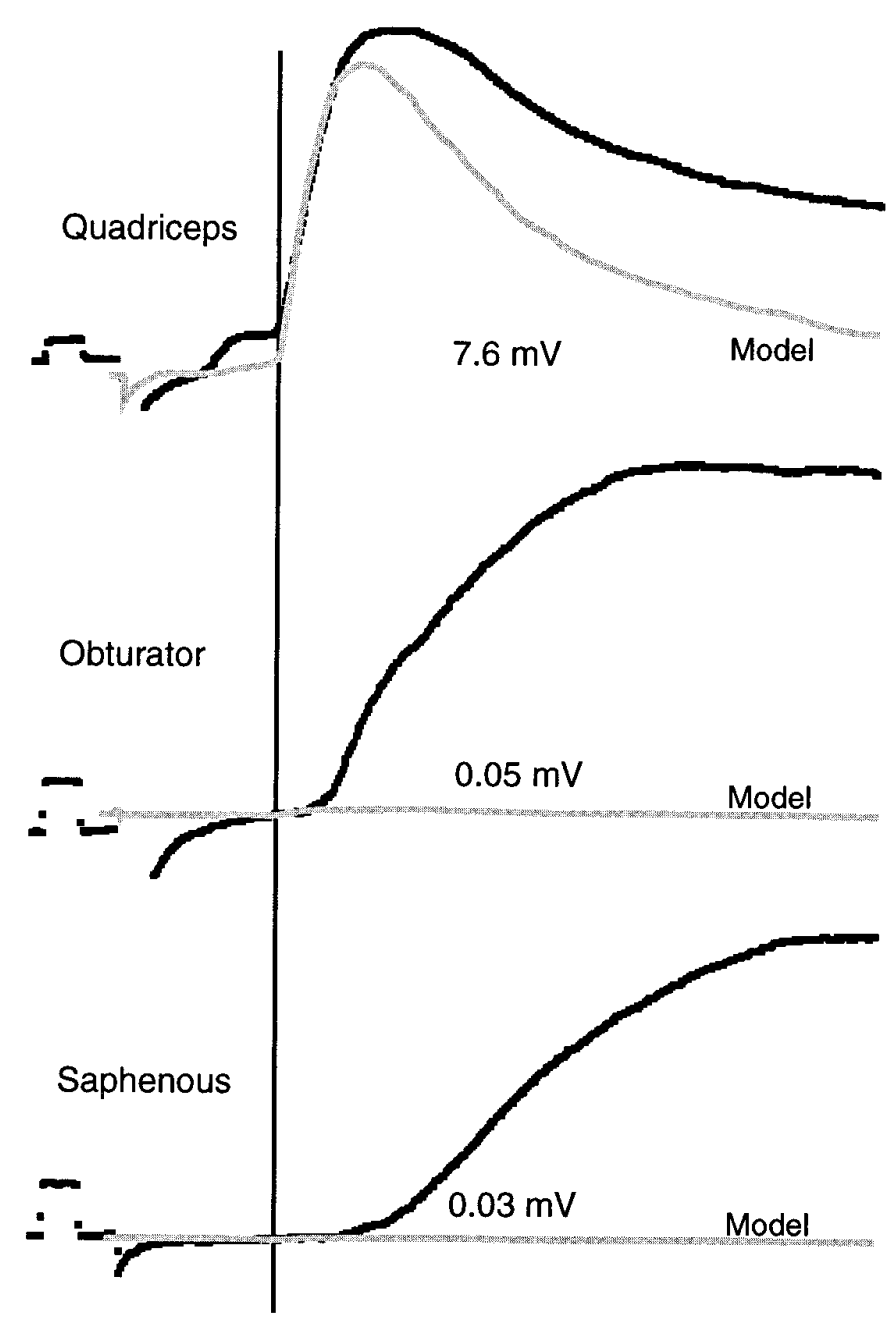

Figure 1. Analysis of synaptic responses with a monosynaptic model. The three examples show three different inputs to a single quadriceps motoneuron in a P5 spinal cord. The top trace shows homonymous input from quadriceps afferents, the middle trace shows input from antagonistic obturator muscle afferents, and the bottom trace shows cutaneous input from afferents in the saphenous nerve. All traces were recorded at $1 \mathrm{~Hz}$ and averaged 60 times. The model trace (Model), shown as a hatched line in each example, was the homonymous input to another quadriceps motoneuron in the same preparation. For each trace the model is scaled so that the first few milliseconds of its rising phase match that of the trace being analyzed. Then the amplitude of the monosynaptic component of the EPSP is taken as the scaled amplitude of the model and is indicated under each trace. Stimulation of the quadriceps nerve evokes a much larger monosynaptic EPSP than from the obturator nerve, although a polysynaptic response evoked by sensory afferents in the obturator nerve begins only 2-3 msec later. The vertical line through all the traces indicates the beginning of the homonymous Ia EPSP in the top and Model traces and is provided to facilitate comparison of the latencies of the three synaptic inputs. A small electrotonic coupling potential from other quadriceps motoneurons precedes the homonymous EPSP; similar coupling potentials are visible preceding some homonymous EPSPs in Figures 3 and 6 . The calibration pulse at the beginning of each trace is $0.5 \mathrm{mV}$ and $2.0 \mathrm{msec}$.

component of the CDRP, which represents the fastest-conducting Ia fibers, was determined as the time between the beginning of the stimulus artifact and the beginning of the upstroke of the first potential. Minimum CDRP latencies for quadriceps and obturator muscle nerves were very similar to each other at each developmental stage; the averages for the two nerves were always within $0.5 \mathrm{msec}$ (data not shown). 


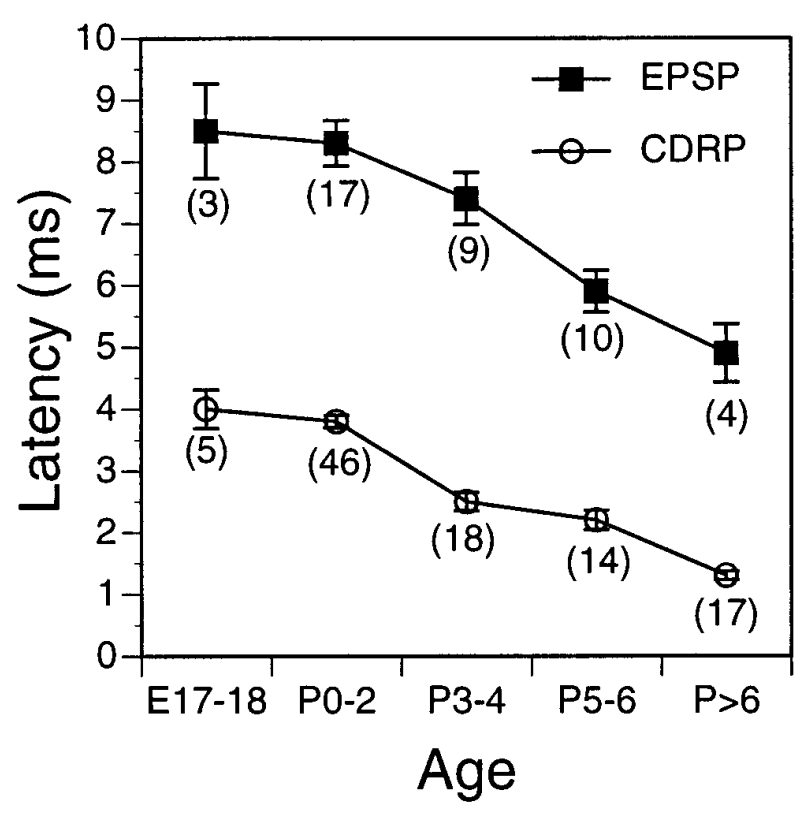

Figure 2. Comparison of latencies of the earliest sensory-motor EPSPs with peripheral conduction times of the most rapidly conducting sensory afferents (cord dorsal root potential, $C D R P$ ) during the first postnatal week. Measurements for homonymous inputs from both the obturator and quadriceps nerves are included for each data point; error bars are \pm 1 SEM. All latencies decrease during development as conduction velocities increase, but the difference in latencies remains relatively constant at $3.5-4.9 \mathrm{msec}$, reflecting the sum of sensory conduction time within the spinal cord plus one synaptic delay. The numbers of recordings for each data point are shown in parentheses.

\section{RESULTS}

\section{Measurement of la monosynaptic inputs to motoneurons}

The major goal in these experiments was to measure accurately the monosynaptic inputs made by Ia sensory neurons onto motoneurons during early postnatal development in the mouse. Conduction velocities of axons change as nerve fibers grow in caliber and become myelinated during the first postnatal week, making it more difficult to assess which synaptic inputs are mediated monosynaptically. To avoid possible contamination from polysynaptic potentials or inputs from other more slowly conducting classes of sensory afferents, we measured the amplitude of only those EPSPs with the shortest latencies. To estimate the central synaptic delay of these potentials, we also measured the peripheral conduction times of the most rapidly conducting sensory axons in muscle nerves.

A comparison of these measurements at several different developmental stages is shown in Figure 2. Both minimal synaptic latencies (i.e., the latencies of the earliest homonymous synaptic potentials) and minimum peripheral conduction times (see Materials and Methods) become shorter as development proceeds and the axons mature. Peripheral conduction time, for example, falls nearly fourfold, from 4 to $\sim 1.1 \mathrm{msec}$. The difference between synaptic latency and peripheral conduction time, however, is relatively constant during this period, falling only $25 \%$ from $\sim 4.7$ msec during the first half week to $3.5 \mathrm{msec}$ at the end of the week. These time differences represent conduction time of the sensory impulses within the spinal cord plus the synaptic delay associated with transmission from sensory afferent terminals to motoneurons. Part of this decrease likely results from a decrease in conduction time within the cord, as in peripheral nerves. The total time available for synaptic transmission therefore changes by, at most, $\sim 1$ msec during the first postnatal week. Given this constancy in synaptic delay and the fact that homonymous Ia-motoneuron connections are known to be monosynaptic in adult animals, EPSPs with these shortest latencies very likely represent monosynaptic input from Ia afferent axons at each time point studied here. Polysynaptic inputs, such as those from some classes of cutaneous afferents, can evoke EPSPs with latencies only 2-4 msec longer than these shortest latencies, as can be seen in the bottom traces in Figure 1. Given the relatively small differences in latency among the different classes of afferent input, it was critical to use a method for measuring EPSPs that discriminated among these classes.

The use of the monosynaptic model, which is described in Materials and Methods, provided such a method. By matching the first few milliseconds of the rising phase of the test EPSP to that of the model, we could estimate the amplitude of the monosynaptic Ia component of the synaptic response. Even large inputs with only slightly longer latencies could be identified unambiguously and excluded. For example, the synaptic potential in the bottom trace of Figure 1 was polysynaptic because it was elicited by stimulation of a cutaneous nerve. Although the first component of this response begins only $3.5 \mathrm{msec}$ later than the homonymous Ia input (top trace), virtually all components were excluded by use of the model trace. In the middle trace of Figure 1, input from afferents supplying an antagonistic muscle, obturator, begins only $2 \mathrm{msec}$ later than the homonymous input, yet use of the model excluded virtually all of it. This slightly later input to antagonist motoneurons was sometimes quite large, as illustrated here, and may represent disynaptic input from Group Ib fibers. These axons supply Golgi tendon organs and are known to provide polysynaptic inputs to a variety of limb motoneurons. Such large polysynaptic inputs from antagonistic muscle afferents emphasize the importance of having a highly restrictive operational definition of Ia monosynaptic input.

\section{The pattern and strength of monosynaptic connections do not change during development}

Amplitudes of monosynaptic Ia EPSPs were analyzed for all four combinations of quadriceps and obturator sensory axons and motoneurons. The pattern of these connections was very similar to that reported for other vertebrate species. Monosynaptic inputs to motoneurons of the same type (homonymous connections) were generally $\sim 10$ times stronger than monosynaptic inputs to antagonistic motoneurons. Representative traces showing these connections near the beginning and end of the developmental period included in this study are shown in Figure 3, and the mean values for each type of connection are shown in Figure 4 as a function of developmental age. At each stage examined, from E17-E18 through the end of the first postnatal week, homonymous connections were prominent and included many relatively large amplitude EPSPs (mean amplitudes $\sim 2 \mathrm{mV}$ ), whereas monosynaptic Ia inputs from antagonistic muscle nerves, in contrast, were small (mean amplitudes $\sim 0.2 \mathrm{mV}$ ). The mean amplitudes of Ia EPSPs correlated highly with the type of sensory neuron and motoneuron (ANOVA: $\left.F_{(1)}=117.6, p<0.0001\right)$, but not with developmental age $\left(F_{(4)}=1.15, p>0.33\right)$.

Because many homonymous connections had been made already by $\mathrm{P} 0$, these results leave open the possibility that larger numbers of inappropriate connections exist during earlier (prenatal) stages of synaptogenesis by Ia axons. Technical difficulties, 


\section{Muscle Nerve Stimulated}

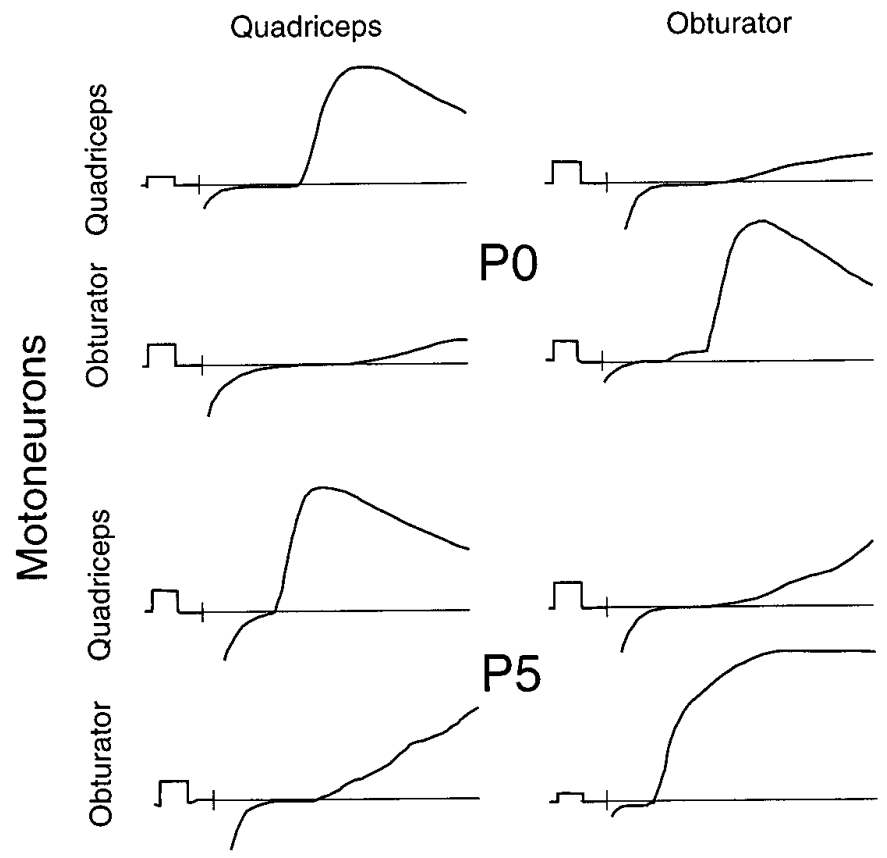

Figure 3. Representative EPSPs in quadriceps and obturator motoneurons elicited by stimulation of afferents in the quadriceps or obturator muscle nerves. The top four traces show recordings from two motoneurons in a $P O$ preparation, whereas the bottom four traces show the analogous connections at P5. Homonymous monosynaptic Ia inputs are relatively large, whereas short-latency inputs from muscle afferents supplying an antagonistic muscle are weak. This pattern of connectivity remains constant throughout the first postnatal week. The beginning of the stimulus is indicated with a short vertical line, and the baseline is indicated with a thin horizontal line. Calibration pulses at the beginning of each trace are 0.5 $\mathrm{mV}$ and $2.0 \mathrm{msec}$.

probably arising from the small size of motoneurons, precluded studying stages earlier than E17, and even at E17-E18 the number of successful recordings was small. Nevertheless, a strong case that many synapses still were being formed during the time period included in this study can be made by examining the distribution of homonymous EPSP amplitudes. Amplitude histograms for each of the four types of connections at each of the five stages studied are presented in Figure 5. From P0 to P4, 8 of $51(16 \%)$ quadriceps motoneurons had $<0.2 \mathrm{mV}$ of homonymous (i.e., quadriceps) Ia input (top row of Fig. 5), yet in adults virtually all motoneurons receive a robust homonymous input (Eccles et al., 1957; Mendell and Henneman, 1971). A likely explanation for the absence of homonymous input to some motoneurons just after birth is that many Ia-to-motoneuron connections still are being made during this period. Despite this ongoing formation of new connections, monosynaptic inputs from antagonistic Ia afferents are small.

Another important point that can be derived from the amplitude histograms in Figure 5 is that a few "inappropriate" connections do exist. Although the average input from antagonistic Ia afferents was $\sim 0.2 \mathrm{mV}$ at each stage, we did record a number of EPSPs larger than $0.6 \mathrm{mV}$, and six were $>1.0 \mathrm{mV}$. However, the incidence of these occasional larger inputs did not change significantly during the first postnatal week. From E17 to P2, 3 of 83 $(3.6 \%)$ antagonist EPSPs were larger than $0.6 \mathrm{mV}$, whereas from P5 onward the incidence of the larger inputs was 4 of $85(4.7 \%)$.
The preference of Ia afferents for homonymous versus antagonistic motoneurons is strong, approximately 10:1 for the connections studied here, but it is not absolute. The critical point in the present context, however, is that the incidence of monosynaptic Ia projections to antagonistic motoneurons does not change as these reflex connections develop.

\section{Effects of stimulation frequency on patterns of connectivity}

Immature mammalian sensory-motor synapses are highly sensitive to repetitive stimulation; even $1 \mathrm{~Hz}$ stimulation can cause significant depression of Ia EPSPs in motoneurons (Lev-Tov and Pinco, 1992; Seebach and Ziskind-Conhaim, 1994). It is possible that by stimulating at $1 \mathrm{~Hz}$ we had selectively fatigued a population of connections that were inappropriate and were eliminated selectively during the first postnatal week. To investigate this possibility, we repeated the experiments, using stimulation frequencies of both 0.1 and $1.0 \mathrm{~Hz}$. First, the amplitudes of the earliest component of the CDRPs were measured at these two frequencies to make sure the same number of rapidly conducting sensory axons was stimulated. The amplitudes at the two frequencies differed by $<10 \%$ at each stage (data not shown), making it very unlikely that possible changes in EPSP amplitude would be caused by changes in the number of axons stimulated.

Intracellular recordings from motoneurons confirmed earlier reports that homonymous short-latency EPSPs were often larger at lower frequencies. This can be seen qualitatively in the representative traces in Figure 6 and more quantitatively in the combined data shown in Figure 7. The increase seems to be smaller than that reported by Lev-Tov et al. (1992), perhaps because we used a bath temperature of $30^{\circ} \mathrm{C}$ instead of $21-24^{\circ} \mathrm{C}$, as in the earlier studies. The warmer temperature may allow synapses to replenish stores of neurotransmitter more rapidly. Synaptic potentials of longer latency were increased more dramatically, as can be seen in the greater rates-of-rise of these potentials in Figure 6, but we did not measure the magnitude of these later inputs.

Despite these increases in EPSP amplitudes, however, the slower stimulation frequency did not reveal a class of short-latency inappropriate inputs that had been overlooked in the first set of experiments. Short-latency inputs from antagonistic muscle nerves (Obt. inputs in top panel and Quad. inputs in bottom panel of Fig. 7) remained at least several-fold smaller than the corresponding homonymous inputs at every developmental stage. At most stages the mean monosynaptic Ia inputs from antagonistic muscles were $<0.3 \mathrm{mV}$, nearly 10 times smaller than the corresponding homonymous inputs. At P5-P6, the inputs from obturator to quadriceps were higher than in the first set of experiments, but the amplitudes were not significantly different at the two frequencies. Overall, stimulation frequency did not correlate significantly with EPSP amplitude in any of the groups of EPSPs (ANOVA: $\left.F_{(3)}=0.029, p>0.99\right)$.

\section{DISCUSSION}

In these experiments we compared the strengths of synaptic connections between sensory and motor neurons supplying the obturator and quadriceps muscles. The motoneurons innervating these muscles are in the same segment of spinal cord (McHanwell and Biscoe, 1981), and the central arbors of their Ia sensory axons overlap (Rivero-Melián, 1996). The situation is similar, in this respect, to lateral geniculate inputs from the two eyes converging on the same localized region of visual cortex and should have 
Figure 4. Mean amplitudes of monosynaptic Ia EPSPs in quadriceps and obturator motoneurons at different developmental ages. The amplitudes (error bars $=1 \mathrm{SE}$ ) for each type of sensory-motor pair are shown for each age group. Each symbol represents inputs from one muscle nerve to one type of motoneuron. For example, Quad. to Obt. represents inputs from the quadriceps nerve to obturator motoneurons. The numbers of pairs examined for each point are shown on the histograms in Figure 5. EPSP amplitudes remain relatively constant during the first postnatal week for all four types of synaptic connection, and there is little input from antagonistic Ia afferents at any stage.

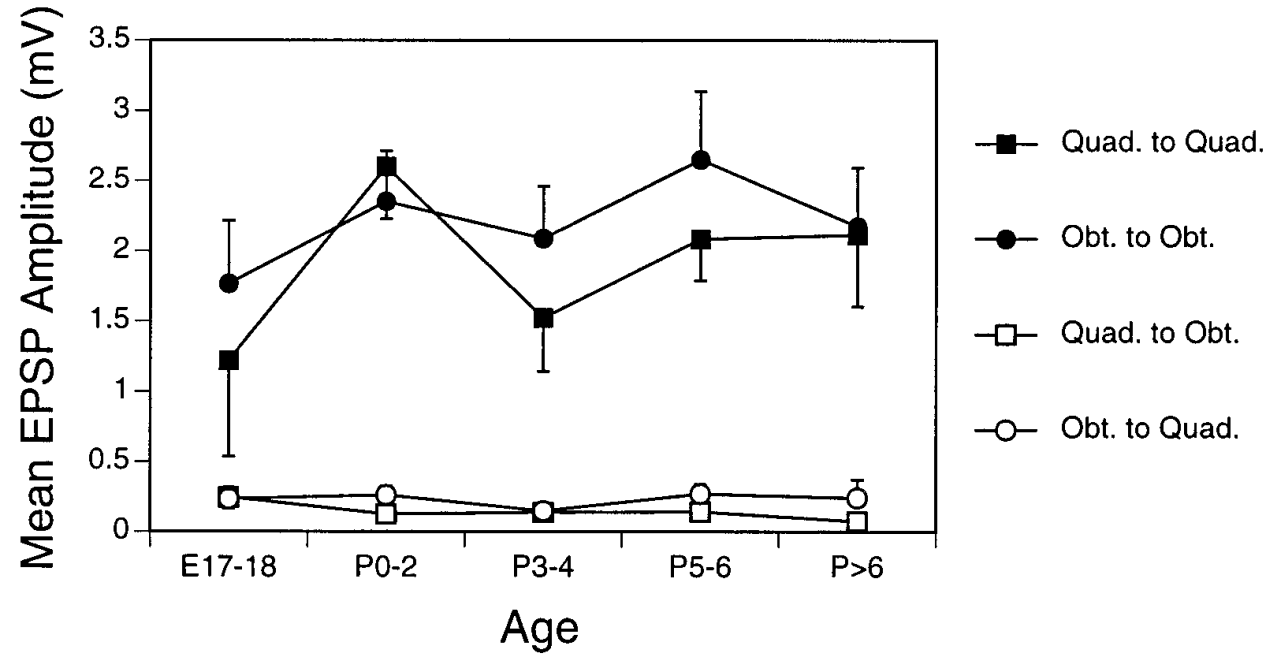

Figure 5. Amplitude histograms of monosynaptic Ia EPSPs in quadriceps and obturator motoneurons at different developmental ages. EPSP amplitudes are divided into $0.2 \mathrm{mV}$ bins, and the $x$-axis scale, indicated for the histogram on the bottom right, is the same for each histogram. The extreme right bin in each histogram includes all responses $>5 \mathrm{mV}$. The scale for the $y$-axes is different for different histograms, but in each case the sum of all columns is the total number of occurrences in that histogram, which is indicated in parentheses. Homonymous inputs are, on average, larger than antagonistic ones at every stage examined, although there are occasional antagonistic EPSPs $>1 \mathrm{mV}$ throughout the first postnatal week.

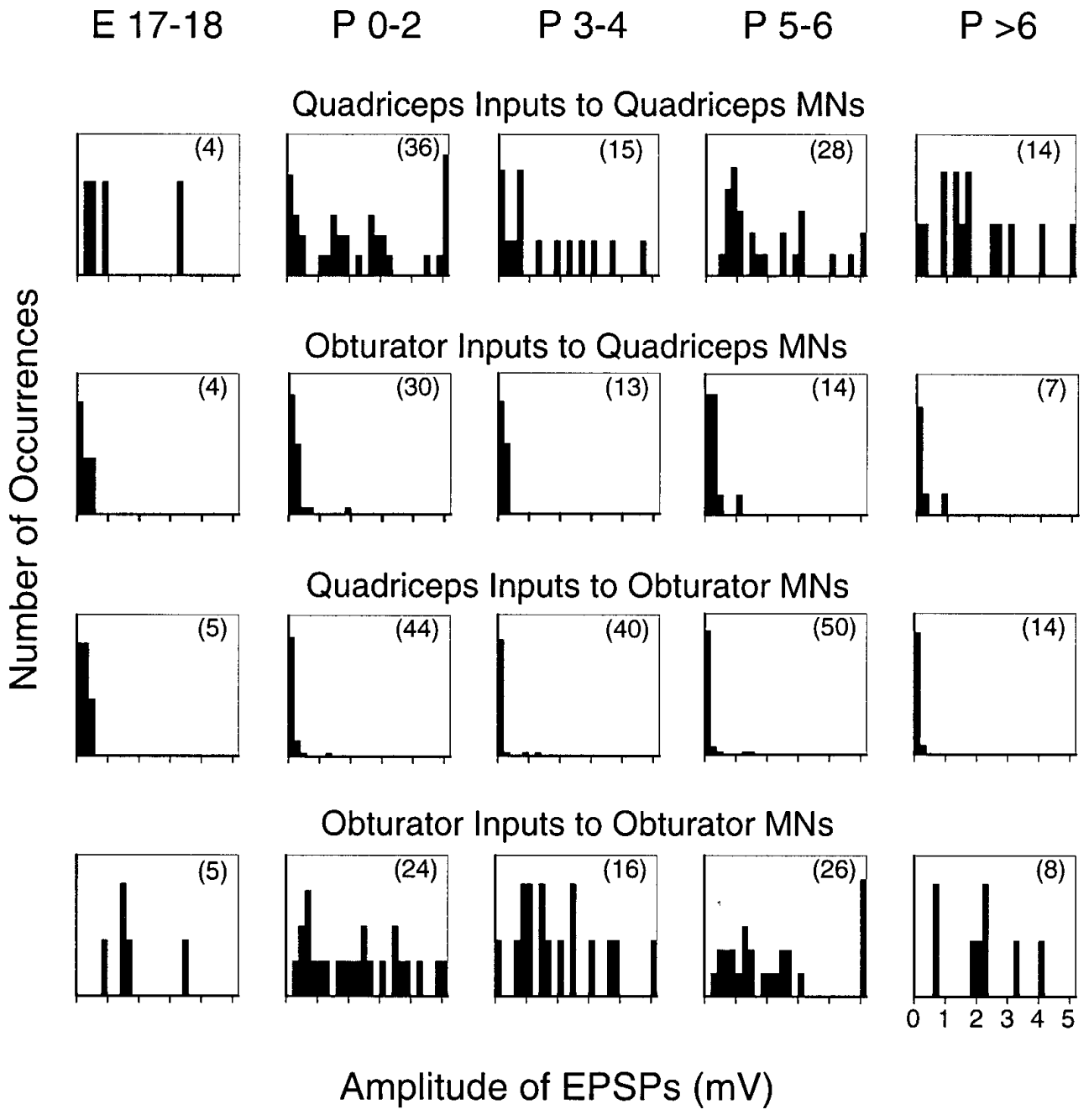

data reported here are therefore unlikely to include polysynaptic inputs.

Our major finding is that the pattern of monosynaptic inputs from Ia afferents to motoneurons did not change from birth until the end of the first postnatal week. Homonymous EPSPs in both obturator and quadriceps motoneurons were $\sim 10$ times larger than the reciprocal "inappropriate" connections between dissim- a monosynaptic model enabled us to discriminate responses beginning only 2-4 msec later than monosynaptic Ia inputs. The increased the chances for observing inappropriate connections that might be eliminated.

It was important to measure only the monosynaptic responses evoked by Ia afferents in motoneurons, because polysynaptic pathways may be established via different mechanisms. The use of 


\section{Muscle Nerve Stimulated}

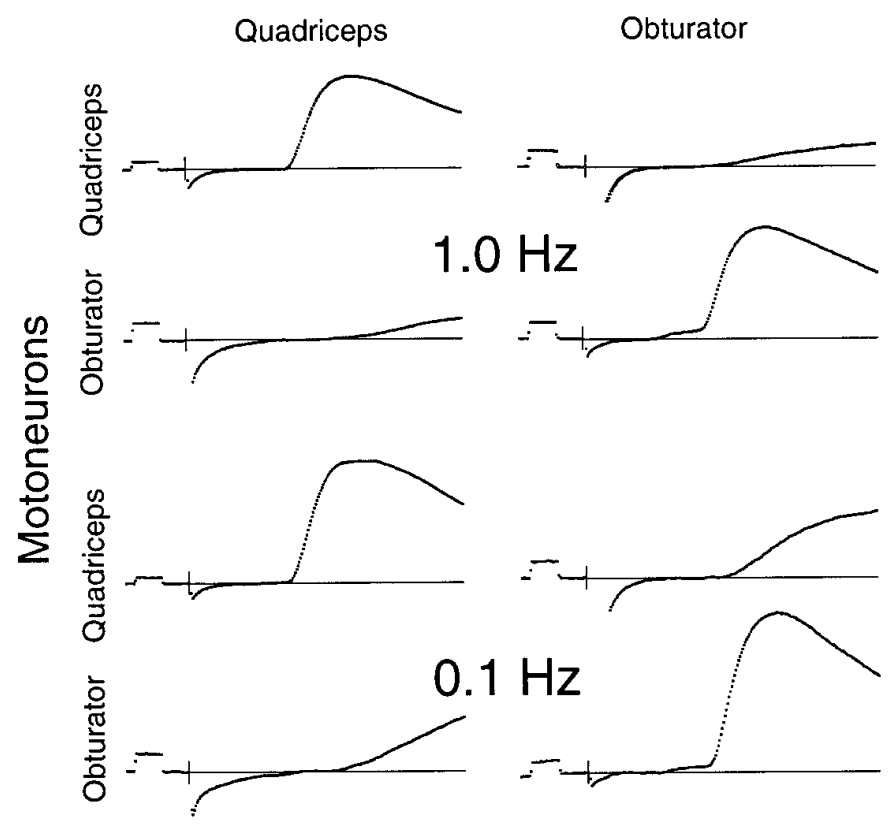

Figure 6. Effects of stimulus frequency on amplitudes of sensory-motor EPSPs. The top traces show homonymous and antagonistic muscle sensory inputs to two motoneurons at $1.0 \mathrm{~Hz}$ stimulation, whereas the bottom traces show the same inputs to the same motoneurons but at $0.1 \mathrm{~Hz}$ stimulation. In no case did stimulation at the lower frequency reveal a significant short-latency input from antagonistic muscle afferents that would have been overlooked at $1.0 \mathrm{~Hz}$. Most synaptic inputs were larger at the lower frequency, although the pattern of connectivity was unchanged. The beginning of the stimulus is indicated with a short vertical line, and the baseline is indicated with a thin horizontal line. Calibration pulses at the beginning of each trace are $0.5 \mathrm{mV}$ and $2.0 \mathrm{msec}$.

ilar sensory and motor neurons at each time point. Nor were inappropriate connections missed because they were easily fatigued by repetitive stimulation. A second series of experiments comparing EPSPs evoked at 0.1 versus $1.0 \mathrm{~Hz}$ found no evidence for a subpopulation of fatigable inappropriate synapses that were eliminated later. Because the pattern we observed at the end of the first postnatal week is similar to the adult pattern in other mammalian species (Eccles et al., 1957), it is likely that there is minimal rearrangement of these connections from the time of birth until adulthood.

The pattern of Ia inputs to motoneurons was not studied at the very earliest stages of synaptogenesis because it was difficult to make reliable intracellular recordings before birth. Axon collaterals from muscle afferents overlap with dendrites of motoneurons by E15.5 (E. Frank, unpublished observations), so a few synapses already may be functional by this stage. Ia inputs to antagonistic motoneurons might, therefore, be more common prenatally. Indirect evidence (see Results) suggests that Ia afferents continue to make synapses with motoneurons during the first postnatal week, however. If appreciable numbers of errors were made during this period, they should have been detected. Moreover, in most systems in which synaptic rearrangements are known to occur, it takes days or even weeks for the pattern of connections to change (for review, see Goodman and Shatz, 1993). If inappropriate monosynaptic connections between Ia afferents and motoneurons are present in the developing spinal cord, they must be relatively short-lived, because they are not apparent by birth,
$<4 \mathrm{~d}$ after these afferents are in an anatomical position to begin making contacts with motoneuronal dendrites.

Changes in the pattern of connections between muscle sensory and motor neurons during postnatal development have been reported in other systems, in contrast to the present results, and it is important to determine why the results are different. In human infants, for example, stimulation of muscle afferents by stretching the soleus tendon elicits short-latency EMG responses in several different leg muscles (Myklebust and Gottlieb, 1993), yet in adults, EMG responses to activation of soleus afferents are much more restricted, implying a substantial change in connections. An important difference between these results and our own is the long peripheral conduction times of sensory and motor axons in humans. Because of this long delay, a distinction between monosynaptic versus di- or even trisynaptic inputs from these or other muscle afferents would be difficult to resolve. Although the nature of these changes will be interesting to explore further, it is not possible to conclude at present that they represent changes in monosynaptic connections.

A study of the development of these connections in neonatal rats by Seebach and Ziskind-Conhaim (1994) is more directly comparable to our own. Using intracellular recordings from ankle flexor and extensor motoneurons in an isolated preparation of the spinal cord, they found a substantial change in the pattern of short-latency muscle afferent input during the first postnatal week. The incidence of inputs from antagonistic muscle nerves dropped from $41 \%$ at $\mathrm{P} 0-\mathrm{P} 2$ to $12 \%$ at $\mathrm{P} 3-\mathrm{P} 5$. Although it is possible that the mechanisms for establishing reflex specificity in rats versus mice or in motoneurons supplying ankle versus thigh muscles are different, it is instructive to look for other possible explanations of the different results.

One important difference between the two studies is that Seebach and Ziskind-Conhaim (1994) did not analyze the amplitudes of inappropriate EPSPs, only their frequencies of occurrence. Any synaptic potential above the noise level presumably was counted if it met the other criteria for being monosynaptic. This makes it difficult to know whether the strength of inappropriate inputs changed during postnatal development. In our own experiments, although the average monosynaptic input from antagonistic muscle nerves was $\sim 0.2 \mathrm{mV}$, there were several cases at each stage in which the input was larger. Our conclusion that synaptic specificity is appropriate from birth is based on the observation that the strength of inputs from antagonistic muscle nerves did not change over time. If the average amplitudes of inappropriate inputs in neonatal rats were also constant over time, it would alter the interpretation that these connections are rearranged.

A second difference in the methods used by Seebach and Ziskind-Conhaim (1994) is that the bath temperature was cooler (21-24 vs $30^{\circ} \mathrm{C}$ in our experiments) and peripheral nerves were longer, resulting in long and variable peripheral conduction times. Antidromic latencies of motoneurons ranged between 10 and 30 msec. There was probably a similar variability in conduction times of sensory axons, although these were not reported. In the absence of knowledge of sensory conduction times, Seebach and Ziskind-Conhaim (1994) defined synaptic delay as the difference in latency between a synaptic potential and the antidromic action potential of the motoneuron under study. Because of variability in the antidromic latencies of different motoneurons, even within a single preparation, situations could occur in which a polysynaptic EPSP could be classified mistakenly as monosynaptic because of its short synaptic delay. As in our experiments, Seebach and Ziskind-Conhaim (1994) found that polysynaptic potentials (for 


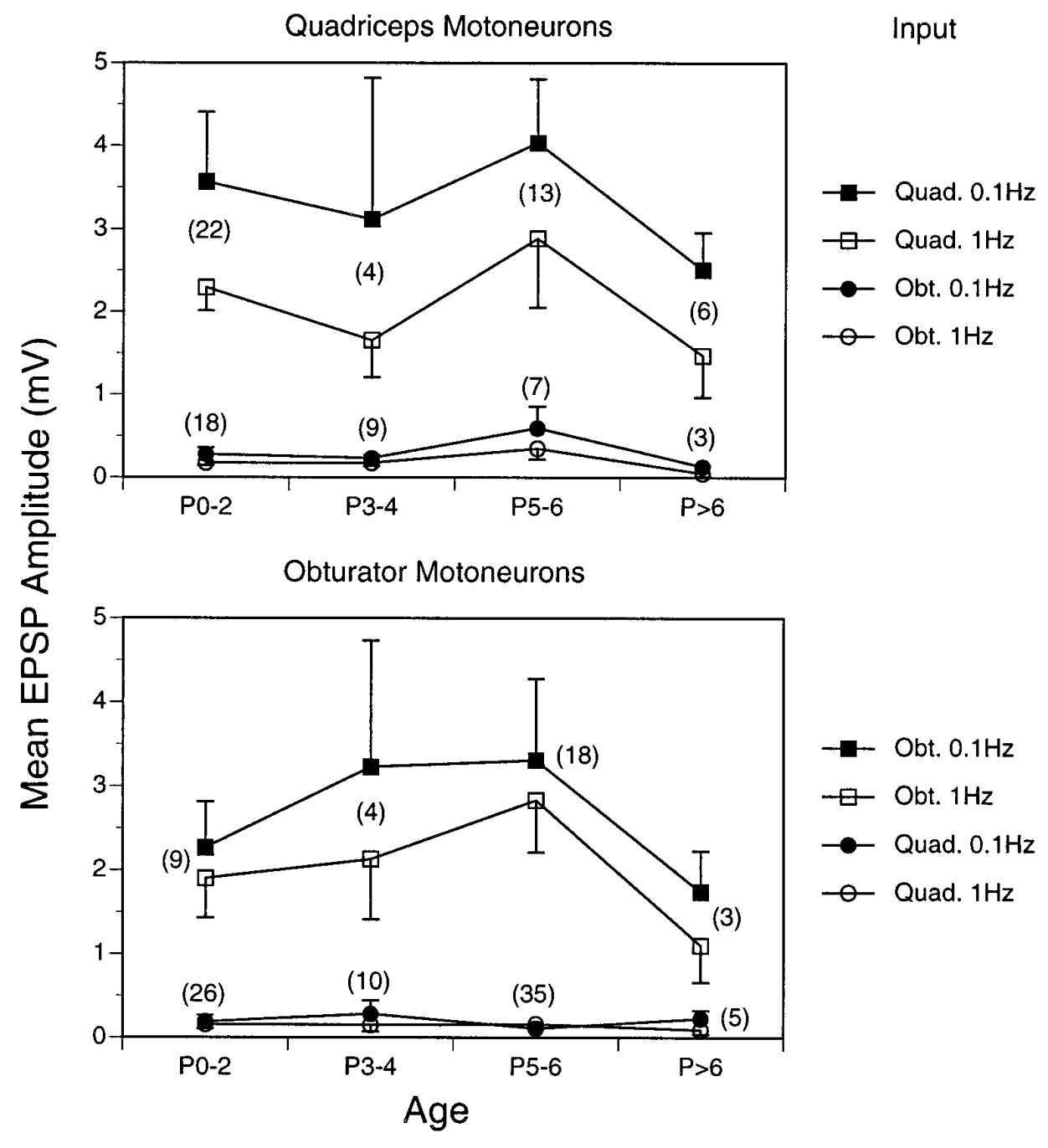

Figure 7. Effect of repetitive stimulation on the amplitude of monosynaptic Ia EPSPs at different times during the first postnatal week. The top and bottom panels illustrate inputs to quadriceps and obturator motoneurons, respectively. The mean monosynaptic amplitudes (error bars $=1 \mathrm{SE}$ ) for each type of sensory-motor pair are shown for each age group. Abbreviations are the same as in Figure 4 . The numbers of pairs examined for each comparison are shown in parentheses.

example, IPSPs) often had only slightly (sometimes only 3-4 msec) longer latencies than monosynaptic ones. With only small differences in latencies, it is critical to measure the actual conduction times of the sensory afferents and to use the same absolute latency (rather than the difference between EPSP and antidromic latency) for classifying potentials as monosynaptic. Although Seebach and Ziskind-Conhaim (1994) make the important point that the synaptic delays of inappropriate inputs were no greater than those of appropriate ones, an unknown fraction of both appropriate and inappropriate inputs in their study may have been mediated polysynaptically.

Another effect of the cooler bath temperatures used by Seebach and Ziskind-Conhaim (1994) was that synaptic potentials often showed pronounced fatigue with repetitive stimulation. Because they found that polysynaptic potentials fatigued more easily than monosynaptic ones, they used resistance to fatigue as an additional criterion for monosynaptic inputs. In the present study the amplitudes of inappropriate inputs were equally low at 1.0 and 0.1 $\mathrm{Hz}$, so inclusion of this additional criterion would not have changed our results.

A limitation in our method for measuring EPSPs is that it is insensitive to monosynaptic inputs from sensory fibers with slower conduction velocities. Group II muscle afferents also supply muscle spindles and provide monosynaptic input to motoneurons (Kirkwood and Sears, 1974). Similarly, less mature Ia afferents would have longer conduction times because they are smaller and less heavily myelinated. Because the EPSPs evoked by both types of afferent would have longer latencies, we would have excluded them. Despite this limitation, however, slower afferents are unlikely to be responsible for the differences in specificity seen in rats versus mice. As mentioned above, the appropriate and inappropriate inputs seen in rats had similarly short synaptic delays.

Given the possibility that some EPSPs reported by Seebach and Ziskind-Conhaim (1994) may have been mediated polysynaptically, we suggest a likely interpretation of both sets of results is that most monosynaptic Ia inputs to motoneurons are appropriate from the outset. According to this view, Ia afferents initially form connections with their correct synaptic partners, and the pattern of these connections remains fixed during postnatal development. The situation in mammals, then, would be similar to that reported for the analogous connections in lower vertebrates. Polysynaptic components of this reflex arc, in contrast, could undergo rearrangements, as suggested by results both in rats and human infants. In this scenario the mammalian stretch reflex is complex, composed of multiple circuits that develop in different manners. Polysynaptic circuits could be rearranged during development, perhaps in an activity-dependent manner, whereas the monosynaptic stretch reflex develops in a evolutionarily conserved manner wherein sensory neurons are directed chemically by their target muscles to form appropriate connections from the outset. 


\section{REFERENCES}

Eccles JC, Eccles RM, Lundberg A (1957) The convergence of monosynaptic excitatory afferents onto many different species of alpha motoneurones. J Physiol (Lond) 137:22-50.

Eccles RM, Shealy CN, Willis WD (1963) Patterns of innervation of kitten motoneurones. J Physiol (Lond) 165:392-402.

Frank E, Jackson PC (1986) Normal electrical activity is not required for the formation of specific sensory-motor synapses. Brain Res 378:147-151.

Frank E, Westerfield M (1982) Synaptic organization of sensory and motor neurones innervating triceps brachii muscles in the bullfrog. J Physiol (Lond) 324:479-494.

Frank E, Westerfield M (1983) Development of sensory-motor synapses in the spinal cord of the frog. J Physiol (Lond) 343:593-610.

Goodman CS, Shatz CJ (1993) Developmental mechanisms that generate precise patterns of neuronal connectivity. Neuron 10:77-98.

Kirkwood PA, Sears TA (1974) Monosynaptic excitation of motoneurones from secondary endings of muscle spindles. Nature 252:243-244.

Lee MT, Koebbe MJ, O’Donovan MJ (1988) The development of sensorimotor synaptic connections in the lumbosacral cord of the chick embryo. J Neurosci 8:2530-2543.

Lev-Tov A, Pinco M (1992) In vitro studies of prolonged synaptic depression in the neonatal rat spinal cord. J Physiol (Lond) 447:149-169.

McHanwell S, Biscoe TJ (1981) The localization of motoneurons supply- ing the hindlimb muscles of the mouse. Proc R Soc Lond [Biol] 293:477-508.

Mendell LM, Henneman E (1971) Terminals of single Ia fibers: location, density, and distribution within a pool of 300 homogeneous motoneurons. J Neurophysiol 34:171-187.

Mendelson B, Frank E (1991) Specific monosynaptic sensory-motor connections in the chick embryonic spinal cord form in the absence of patterned neural activity and motoneuronal cell death. J Neurosci 11:1390-1403.

Myklebust BM, Gottlieb GL (1993) Development of the stretch reflex in the newborn: reciprocal excitation and reflex irradiation. Child Dev 64:1036-1045.

Naka K (1964a) Electrophysiology of the fetal spinal cord. I. Action potentials of the motoneuron. J Gen Physiol 47:1003-1022.

Naka K (1964b) Electrophysiology of the fetal spinal cord. II. Interaction among peripheral inputs and recurrent inhibition. J Gen Physiol 47:1023-1038.

Rivero-Melián C (1996) Organization of hindlimb nerve projections to the rat spinal cord: a choleragenoid horseradish peroxidase study. J Comp Neurol 364:651-663.

Sah DWY, Frank E (1984) Regeneration of sensory-motor synapses in the spinal cord of the bullfrog. J Neurosci 4:2784-2791.

Seebach BS, Ziskind-Conhaim L (1994) Formation of transient inappropriate sensorimotor synapses in developing rat spinal cords. J Neurosci $14: 4520-4528$. 